\title{
Kernos
}

Revue internationale et pluridisciplinaire de religion grecque antique

6 | 1993

Varia

\section{L'Iynge dans le discours mythique et les procédures magiques}

\section{Vinciane Pirenne-Delforge}

\section{(2) OpenEdition \\ Journals}

\section{Édition électronique}

URL : http://journals.openedition.org/kernos/552

DOI : 10.4000/kernos.552

ISSN : 2034-7871

\section{Éditeur}

Centre international d'étude de la religion grecque antique

Édition imprimée

Date de publication : 1 janvier 1993

Pagination : 277-289

ISSN : 0776-3824

\section{Référence électronique}

Vinciane Pirenne-Delforge, "L'lynge dans le discours mythique et les procédures magiques », Kernos [En ligne], 6| 1993, mis en ligne le 07 avril 2011, consulté le 01 mai 2019. URL : http:// journals.openedition.org/kernos/552; DOI : 10.4000/kernos.552 
Kernos, 6 (1993), p. 277-289.

\section{L'TYNGE DANS LE DISCOURS MYTHIQUE} ET LES PROCÉDURES MAGIQUES*

Dans le domaine des sentiments et des attirances amoureuses, quand la contrainte se substitue à la persuasion, intervient soit la force directe, brutale, soit l'action magique ${ }^{1}$. Les Grecs étaient particulièrement sensibles à la primauté de $\pi \varepsilon i \theta \omega$ sur $\beta i ́ \alpha$ dans les relations entre citoyens sur l'agora et entre époux dans la vie conjugale. C'est en tout cas ce qu'une recherche sur les cultes de Peitho a révélé, tout autant qu'une analyse des interventions de la persuasion sur la scène tragique $^{2}$. Les Suppliantes d'Eschyle, de même que le superbe fragment des Danaïdes où Aphrodite exalte la puissance de l'amour ${ }^{3}$, sont de parfaites illustrations des bienfaits de $\pi \varepsilon i \theta \omega$ dans les relations humaines qui, sans elle, offrent le spectacle désolé d'un désastre, à l'instar des noces assassines des filles de Danaos.

Cette étude entend montrer par quels moyens, quels modes d'expression les Grecs, essentiellement dans le discours mythique, présentaient le sentiment amoureux, le désir entre les êtres. En poursuivant cette enquête jusqu'aux extrémités que donne au thème son expression magique, on espère donner, sur ce point précis, quelques indications sur les relations problématiques de la religion et de la magie ${ }^{4}$.

* Cet article est la version remaniée d'un exposé présenté à l'Université de Valladolid, dans le cadre d'un colloque intitulé Magia y mantica en la Grecia antigua y en Bizancio. Je tiens à remercier le professeur Emilio SUÁREZ DE LA TORRE de son invitation.

1 Sur l'application de procédés de magie incantatoire dans le domaine amoureux, cf. J.J. WINKLER, The Constraints of Eros, in Christopher A. FARAONe, Dirk ObBINK (éds), Magika Hiera. Ancient Greek Magic \& Religion, New York, Oxford University Press, 1991, p. 214-243.

2 V. Pirenne-Delforge, Le culte de la persuasion. Peithô en Grèce ancienne, in $R H R, 208$ (1991), p. 395-413. Sur la notion d'üßpı dans le droit athénien pour stigmatiser la violence sexuelle, cf. D. COHEN, Sexuality, Violence, and the Athenian Law of Hubris, in $G \& R, 38$ (1991), p. 171-188.

3 ESCH., fr. 44 Nauck $^{2}$, cité par A THÉNÉE, XIII, $600 \mathrm{~b}$.

4 S'attaquer à un thème relevant de ce que nous avons coutume d'appeler "magie» n'est pas sans poser des problèmes de méthode et de définitions. 
Trois grands textes archaïques offrent une assise sûre et très riche à l'exposé : l'épisode de la "tromperie de Zeus" par Héra dans l'Iliade, celui de la création de Pandore dans Les Travaux et les Jours d'Hésiode et l'entreprise de séduction d'Anchise par Aphrodite dans l'Hymne homérique en l'honneur de la déesse. Les thèmes de la séduction et du désir y sont récurrents et l'expression mythique qu'ils revêtent permet de cerner les modes d'action spécifiques d'Aphrodite, la divinité tout particulièrement concernée par ces questions.

\section{Un ruban magique}

Au livre XIV de l'Iliade, Héra entreprend de séduire son époux afin de détourner son attention du champ de bataille et, en endormant sa méfiance, donner l'avantage aux Achéens ${ }^{5}$. Pour ce faire, la déesse prend grand soin de son apparence. Ses apprêts sont décrits avec minutie et aucune étape de la stratégie divine, et avant tout féminine, n'est négligée dans la description du poète. Coiffure, habillement, parfums, bijoux, le harnachement de l'épouse soucieuse de plaire n'a rien à envier à celui d'un soldat prêt à combattre. Cependant, avant de s'avancer vers le roi des dieux, Héra entreprend une démarche supplémentaire : elle sollicite Aphrodite. Prenant prétexte d'un voyage aux confins de l'univers en vue de réconcilier Okéanos et Téthys, Héra

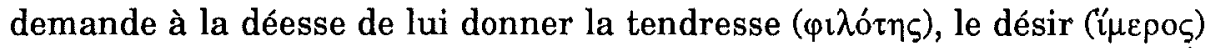
par lesquels elle soumet à la loi de l'amour les dieux et les mortels ${ }^{6}$. Aphrodite accepte et détache de son sein le ruban brodé multicolore

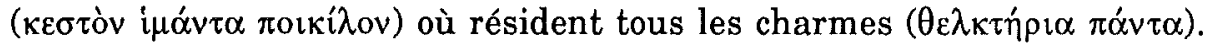

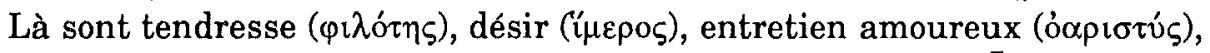
conseil perfide ( $\pi \alpha \alpha_{\rho} \varphi \alpha \sigma \zeta \varsigma$ ) qui trompe le cœur des plus sages?

Depuis longtemps, le débat sur les rapports entre religion et magie est engagé, les prises de positions allant d'un partage strict des deux domaines au refus du terme même de «magie», censé n'être qu'une construction d'auteurs modernes projetée sur des cultures différentes. Cf. H.S. VERSNEL, Some Reflections on the Relationship Magic-Religion, in Numen, 38 (1991), p. 177-197. On n'ambition ne nullement d'apporter une quelconque réponse à ce vaste problème. Plus modestement, il s'agit de confronter diverses représentations grecques de la naissance du désir, dans ses composantes que l'on peut qualifier, par commodité, de religieuses et de magiques.

5 Hом., Il., XIV, 153-351.

6 Ibid, $197-210$.

7 Ibid, 211-217. 
Les atouts de la séduction sont en quelque sorte matérialisés dans un des éléments de son habillement. Quelques modernes se sont penchés sur la nature de ce "ruban", de cette "lanière" ${ }^{8}$. On a proposé d'y reconnaître un pectoral ou un ruban croisé sur la poitrine et dans le dos. Par analogie avec le terme d'héraldique désignant la croix de saint André, le nom de "sautoir» lui a été attribué. Ce rapprochement trouve sa source dans les représentations de déesses orientales qui portent fréquemment un tel attribut ${ }^{9}$. L'adjectif кєơós laisse penser que l'objet était brodé ou, selon une autre interprétation étymologique du mot, décoré à l'aide de petits trous ${ }^{10}$. Quel que soit l'objet exact que l'aède a voulu évoquer, il semble en tout cas que la détermination de sa fonction est bien plus essentielle pour la compréhension du passage que la définition précise de sa nature. En effet, le mot ï $\mu_{\varsigma} \varsigma$ qui sert à le désigner est utilisé quand sont mentionnées, dans l'Iliade, des courroies de casque ou des rênes de chevaux ${ }^{11}$. Il s'agit donc bien d'une lanière, d'un lien qui peut servir à «dompter» un animal, comme Aphrodite, selon les termes mêmes utilisés par Héra, "dompte» mortels et immortels ${ }^{12}$. Il n'est peut-être pas indifférent à cet égard que l'intervention d'Aphrodite, au chant III, pour sauver Pâris mis en grande difficulté par Ménélas fou furieux, consiste à empêcher le roi de Sparte d'étrangler le Troyen au moyen de la cour-

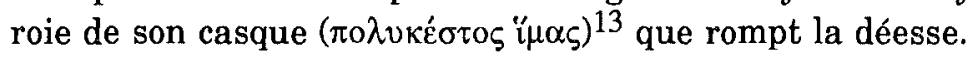

Que retenir dès lors de ces textes ? 1) Aphrodite possède en propre la séduction persuasive que la seule beauté d'Héra ne suffit pas à mettre en œuvre; 2) La ceinture prêtée par Aphrodite offre l'image d'un mode d'intervention spécifique puisqu'elle figure un lien dans lequel la déesse enserre celui qu'elle entreprend de séduire; 3) La ceinture est $\pi \circ{ }^{\prime} i \lambda{ }^{\circ} \varsigma$, chatoyante, multicolore. Nous verrons dans la suite que cette

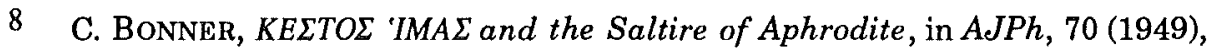
p. 1-6; F.E. B RENK, Aphrodite's Girdle : No Way to Treat a Lady, in CB, 54 (1977), p. 17-19.

9 R. DU MESNIL DU B UISSON, Le sautoir d'Atargatis et la chaîne d'amulettes, Leiden, 1947 (Documenta et Monumenta Orientis Antiqui, I, 1).

10 P. Chantraine, Dictionnaire étym. de la langue grecque, I, Paris, 1968, p. 515, s.v. Kevtéo.

11 Hом., Il., VIII, 543; X, 475; XXIII, 324.

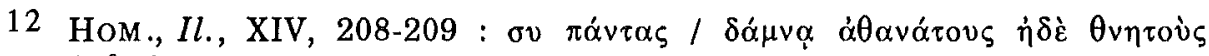

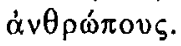

13 III, 371. 
qualité ne relève pas uniquement du critère esthétique, mais que l'épithète apparaît dans des contextes où son aura magique est évidente.

\section{Pour créer une femme}

Ces dernières années, divers travaux de qualité ont mis en lumière le rôle particulier d'Éros et d'Aphrodite dans les unions qui président aux successions divines que décrit la généalogie d'Hésiode ${ }^{14}$. Des unions indifférenciées des origines, on passe, avec la naissance d'Aphrodite, à une différenciation sexuelle marquée par l'introduction

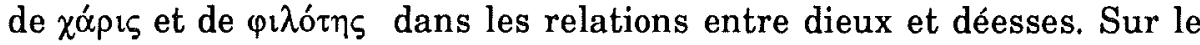
plan humain, c'est la fin de la douce promiscuité originelle avec les dieux qui inaugure, dans le discours hésiodique, une relation spécifique entre les sexes. La création de Pandore, si elle n'introduit pas la femme en tant que telle ${ }^{15}$, définit un type de rapport laissé dans l'ombre avant la crise cosmogonique de Mékonè.

Les Travaux et les Jours présentent à la fois les ordres de Zeus pour le modelage de la femme et la réalisation de ce piège destiné aux humains. «Il commande à l'illustre Héphaïstos de tremper d'eau un peu de terre sans tarder, d'y mettre la voix et les forces d'un être humain et d'en former, à l'image des déesses immortelles, un beau corps aimable de vierge; Athéna lui apprendra ses travaux, le métier qui tisse mille couleurs; Aphrodite d'or sur son front répandra la grâce ( $\chi \alpha$ ópı), le

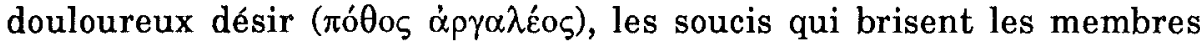

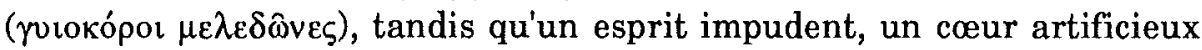
seront, sur l'ordre de Zeus, mis en elle par Hermès, le Messager, tueur d'Argos» ${ }^{16}$. Les dieux obéissent immédiatement et chacun s'attelle à son ouvrage spécifique.

Dans la Théogonie, au moment de la naissance d'Aphrodite, le poète s'était attaché à l'évocation de ses privilèges immémoriaux : les conversations de jeunes filles ( $\pi \alpha p \theta$ évıo óó $\rho \circ)$ ), les sourires ( $\mu \varepsilon i \delta \eta \mu \alpha \tau \alpha)$,

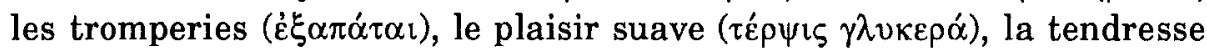

14 Citons entre autres : Annie BonNAFÉ, Eros et Éris. Mariages divins et mythe de succession chez Hésiode, Lyon, 1985; J. RUDHARDT, Le rôle d'Éros et d'Aphrodite dans les cosmogonies grecques, Paris, 1986.

15 Sur ce point, cf. J. RudHARd, Pandora : Hésiode et les femmes, in $M H, 43$ (1986), p. 237-242.

16 HÉsIode, Trav. et Jours, 60-68 (trad. P. Mazon). 
( $\varphi \imath \lambda$ ó $\eta \varsigma)$, la douceur $\left(\mu \varepsilon\left\llcorner\lambda\left\llcorner\chi^{\prime} \mid \alpha\right)^{17}\right.\right.$, ce qui correspond presque textuellement aux "charmes» du ruban brodé de la déesse dans l'Iliade. Chaque poète a présenté à sa manière les prérogatives de la déesse. Pour Homère, il fallait qu'Aphrodite remette concrètement à Héra la matérialisation de ses pouvoirs. Avec Hésiode, l'abstraction est plus poussée.

Dans la description de la création de Pandore, au moment où Aphrodite est censée intervenir, elle n'apparaît plus nommément : autour du cou de Pandore, les Charites et Peitho mettent des colliers d'or, tandis que les Heures la parent de guirlandes de fleurs printanières ${ }^{18}$. Ces bijoux d'or et de fleurs trouvent la source de leur pouvoir dans les qualités des suivantes d'Aphrodite qui les disposent autour de la femme, Charis, Peitho, grâce et persuasion qui susciteront le douloureux désir voulu par Zeus. C'est un véritable réseau de liens disposé sur la femme qui lui confère une séduction irrésistible, tout comme Héra attirait Zeus par les vertus du ruban brodé. Dans l'Hymne homérique à Aphrodite, nous voyons la déesse elle-même soumise à la loi qu'elle édicte volontiers pour les autres. La stratégie de la séduction ressemble à s'y méprendre aux apprêts d'Héra dans l'Iliade, mais cette fois, nulle mention d'un ruban "magique»: Aphrodite personnifie la force contraignante du désir.

Avec les notions de circularité et de liens, nous entrons dans le domaine de l'intelligence rusée, de la mètis, finement dégagée par M. Detienne et J.-P. Vernant ${ }^{19}$, et des principes mis en œuvre dans le domaine des contraintes magiques. Lors de la création de Pandore, les quatre divinités appelées par Zeus pour réaliser son plan, Héphaïstos, Athéna, Aphrodite, Hermès, sont celles qui sont le mieux pourvues, dans le panthéon grec, de ce type particulier d'intelligence, la mètis, faite de ruse, de tromperie et d'une clairvoyance opportune. Le chant de Démodocos dans l'Odyssée ${ }^{20}$, qui met en scène le piège dans lequel Héphaïstos prend les amants Aphrodite et Arès, illustre le savoir-faire du forgeron tout en montrant la déesse de l'amour enchaînée dans un réseau de liens circulaires infrangibles dont, sur un plan métaphorique, elle est le plus souvent l'auteur.

17 HÉsIODE, Théog., 200-206.

18 Ibid, $73-75$.

19 M. Detienne, J.-P. Vernant, Les ruses de l'intelligence. La mètis des Grecs, Paris, 1974, p. 261-304.

20 Ном., Od., VIII, 266-369; surtout 296-299; 317-318; 335-342. 
Cette même notion de circularité et de liens nous situe de plain-pied dans le domaine des procédures magiques, notamment celles qui touchent aux charmes amoureux. Mais avant d'envisager cette question dans la pratique des procédures, voyons ce que nous dit encore le mythe.

\section{L'oiseau du délire}

Dans la quatrième Pythique, chantée en l'honneur d'Arcésilas de Cyrène, Pindare retrace les exploits de Jason à la recherche de la toison $d$ 'or. Aphrodite intervient pour favoriser son entreprise en lui accordant l'amour de Médée, l'auxiliaire indispensable pour échapper aux pièges de la conquête. Mais comment susciter la passion d'une magicienne habile si ce n'est en retournant contre elle les procédures qu'elle affectionne? Et Pindare d'enchaîner ${ }^{21}$ :

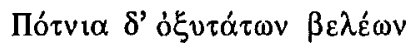

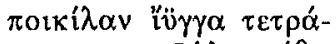

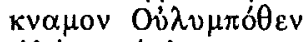

$\dot{\varepsilon} v \dot{\alpha} \lambda \dot{v} \tau \omega \kappa \dot{v} \kappa \lambda \omega$

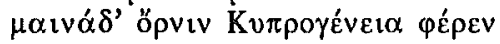

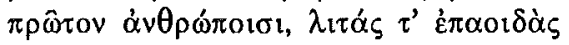

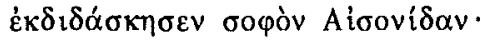

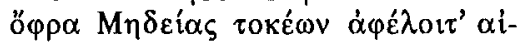

$\delta \hat{\omega}, \pi 0 \theta \varepsilon \imath \dot{\alpha} \delta^{\prime}$ ' $E \lambda \lambda \dot{\alpha} \dot{\alpha}_{\zeta} \alpha \dot{v} \tau \alpha \dot{\alpha} v$

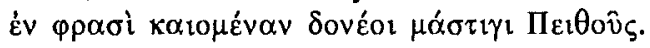

La maîtresse des flèches les plus rapides, la déesse née à Chypre, du haut de l'Olympe, attacha solidement sur une roue le torcol au plumage varié, lié aux quatre membres. Elle apporta, pour la première fois, aux hommes l'oiseau du délire, et enseigna à l'habile fils d'Aison des charmes et des formules, pour qu'il pût faire oublier à Médée le respect de ses parents; pour que le désir de voir la Grèce tourmentât son âme enflammée d'amour et lui fít sentir l'aiguillon de Peitho.

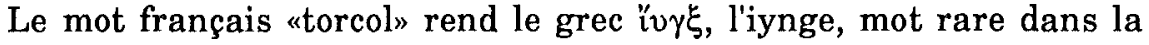
littérature grecque et dont l'utilisation est soit pratique soit métaphorique. D'un point de vue zoologique, Aristote enseigne que l'iynge est un des rares oiseaux à avoir deux doigts en avant et deux doigts en arrière, qu'il est un peu plus grand que le pinson et pousse de petits cris aigus. Ses caractéristiques les plus frappantes sont sa capacité à délier longuement la langue à la manière des serpents et la rotation qu'il peut imprimer à son cou, le reste du corps restant immobile ${ }^{22}$. Remarquable

21 PIND., Pyth., IV, 380-390 (trad. d'après A. Puech).

22 ARISTOTE, Hist. Anim., II, 12 (504a). 
par ses contorsions et ses mouvements incessants, le torcol fait donc son entrée dans l'Olympe par la grâce de la poésie de Pindare. C'est cet

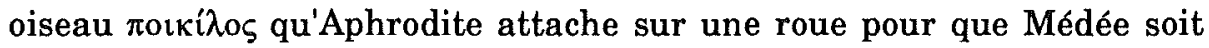
saisie du délire que les Anciens avaient attribué au remuant volatile, lequel doit, avec la force des incantations enseignées à Jason par la déesse, contribuer à tourmenter Médée de l'aiguillon de Peitho. Ce n'est

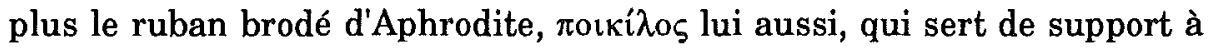
une persuasion amoureuse bien contraignante, mais cet oiseau retenu par des liens à une roue : le mouvement est domestiqué, asservi par les artifices d'une déesse qui concrétise dans l'objet ainsi réalisé les symboles extrêmes de sa capacité à unir les êtres : le cercle et les liens. C'est Pothos et Peitho qui tourmentent Médée, le désir douloureux déjà évoqué à propos de Pandore et la Persuasion tantôt bénéfique tantôt maléfique ${ }^{23}$, présente dans la plupart des textes envisagés ${ }^{24}$.

De la même manière que des dieux ont jadis révélé aux humains des $\tau \varepsilon \chi v \alpha i^{25}$, Aphrodite enseigne à Jason une procédure que l'on s'accordera sans peine à qualifier de "magique»; comme si les armes communes de la divinité et ses modes d'action habituels ne suffisaient pas dans le cas d'une étrangère redoutable par ses artifices.

Les scholies à la IVe Pythique de Pindare révèlent que l'objet créé par Aphrodite possède une consistance historique dans la panoplie des magiciennes et des jeteuses de sort ${ }^{26}$, dont Théocrite, dans sa IIe Idylle, donne un tableau très vivant. Une jeune femme, Simaitha, qui vit seule avec sa servante, entretient une liaison passionnée avec un bel éphèbe du nom de Delphis. Mais les visites de l'amant se font rares, au désespoir de Simaitha qui décide de mettre en œuvre les pouvoirs de la magie dont elle semble connaître quelques expédients. La recette qu'elle applique comporte neuf actions et/ou invocations entrecoupées d'une sorte de refrain où le torcol, l'iynge, est invoqué pour attirer l'infidèle

23 Dualité bien mise en lumière par $\mathrm{M}$. Detienne, Les maîtres de vérité dans la Grèce archä̈que, Paris, 1967, p. 62-66.

24 D'autres auteurs du Ve siècle utilisent le torcol dans leur discours, mais son emploi est alors métaphorique et désigne l'attrait irrésistible que suscite la beauté d'un être ou le charme d'une parole : SOPH., fr. 474 Radt (= ATHÉNÉE, XIII, 564b-c); ARISTOPH., Lys., 1110. Chez EsCHYLe, Perses, 988, c'est le souvenir et le regret de ses compagnons morts au combat qui sont symbolisés, pour Xerxès, par l'iynge. - Cf. aussi Pind., Ném., IV, 56.

25 Eur., Bacchantes, 275-281, par exemple.

26 Schol. Pind., Pyth., IV, 381a Drachmann. 
vers la demeure de la magicienne. A.S.F. Gow, qui a étudié la question à la faveur de son édition de Théocrite, a bien montré que l'iynge du refrain était l'objet de magie amoureuse créé par Aphrodite dans la IVe Pythique de Pindare. La magicienne l'actionnait vraisemblablement entre chaque action ou parole efficace, décrite en quatre vers par le poète ${ }^{27}$. Gow en a également offert quelques représentations anciennes qui permettent de comprendre comment cet instrument fonctionnait. Il s'agit d'une roue - ou parfois d'un disque - à rayons, percée d'au moins deux trous traversés par un fil attaché à ses extrémités. En saisissant dans chaque main une des extrémités de cette lanière circulaire et en lui imposant alternativement traction et relâchement, il est possible de faire tourner la roue rapidement dans un sens puis dans l'autre ${ }^{28}$. Il n'est guère vraisemblable que l'oiseau torcol ait été effectivement entravé aux rayons de la roue, ce que semblent avoir un peu hâtivement conclu les scholiastes de Théocrite ${ }^{29}$, se fondant sans doute sur la description mythique de Pindare. C'est par analogie avec les fréquents mouvements circulaires de l'oiseau que l'instrument rotatif porte son nom ${ }^{30}$. Les capacités peu communes de l'oiseau tout autant que l'objet homonyme devaient être suffisamment connus à l'époque où Pindare décrit la roue du délire puisque l'emploi métaphorique du mot ìv $\xi$ apparaît çà et là dans des œuvres du Ve siècle ${ }^{31}$.

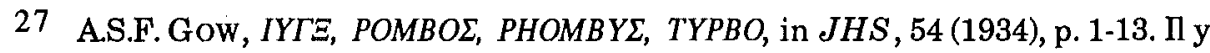

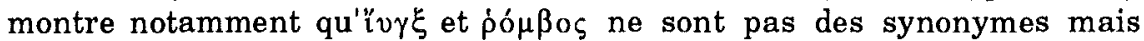
désignent des objets différents.

28 Ibid, p. 3.

29 Schol. ThÉocRITE, Id, II, 17 Wendel. Cf. aussi Photios et la Souda, s. v. "Iv $\gamma \xi$.

30 De surcroît, les Anciens avaient observé qu'il remuait beaucoup la queue et l'avaient donc baptisé du sobriquet de $\sigma \varepsilon 1 \sigma o \pi v \gamma i \varsigma$, "tortille-fesses» en le qualifiant de «débauché» : schol. THÉOCRITE, Id., II, 17; schol. LyCOPHRON,

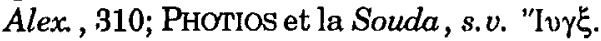

31 Cf. supra, n. 24. L'utilisation se généralisera dans la suite : DIOG. LAËRCE, VI, 76; HÉLIODORE, Ethiopiques, II, 33, 6; IV, 15, 2; VIII, 5,7; HimÉRIUS, 48, 310 Colonna; S YNÉSIUS, Lettres, 146, 4; 41. - Certains auteurs opèrent des rapprochement entre l'iynx et les pouvoirs du ceste d'Aphrodite : HIMÉRIUS, 66, 65; Philostrate Jr., Imagines, 872, 27. 


\section{Iynx et Ixion dans l'Olympe ${ }^{32}$}

Au dire de scholiastes et de lexicographes, Iynx ne fut pas toujours le nom d'un oiseau. Une nymphe portant ce nom, fille d'Écho ${ }^{33}$ ou de Peitho, aurait ensorcelé Zeus lui-même afin qu'il tombe éperdument amoureux d'elle ou d'Io. Ayant encouru de ce fait la terrible et juste colère d'Héra, l'impudente aurait été métamorphosée en cet oiseau qui porte désormais son nom ${ }^{34}$.

Selon le principe des généalogies mythiques en vertu duquel les enfants héritent des pouvoirs de leurs parents en les spécialisant ${ }^{35}$, les mères de la nymphe Iynx situent ses modes d'intervention dans le registre de la parole : lancinante et répétitive dans le cas d'Écho; persuasive et même fourbe avec Peitho. La première symbolise en outre le $\pi$ ó $\theta_{\varsigma}$ dans l'amour puisque sa métamorphose lui permit d'échapper au dieu Pan qui en vain la désira. La seconde, nous l'avons vu, est largement présente dans le cortège d'Aphrodite. C'est donc bien le domaine amoureux qui voit apparaître Iynx et ses sortilèges.

Nombreuses sont les conquêtes de Zeus et, corrélativement, les colères et vengeances d'Héra. Si le dieu se soumet généralement de bonne grâce au joug d'Aphrodite, il ne s'agit pas, dans le cas d'Iynx, d'un doux égarement dicté par le désir mais d'une contrainte ensorcelante qui modifie les règles habituelles du jeu divin de l'adultère. Dans la version qui fait d'Iynx l'objet du désir de Zeus, les rôles sont renversés et le maître de l'Olympe n'a plus l'initiative. Cet état de fait menace encore davantage l'union légitime dont Héra est la garante féroce. Quant à l'intervention alternative d'Io dans ce mythe, elle fait fi des versions habituelles qui font du désir de Zeus pour Io une de ses folâtries parmi d'autres en territoire humain, sans qu'il soit jamais question de

32 Cette partie de l'exposé doit beaucoup à un développement de M. DETIENNE, Les Jardins d'Adonis. La mythologie des aromates en Grèce, Paris, $1989^{2}$ [1972], p. 160-170.

33 CALL., fr. 685 Pfeiffer.

34 Schol. Pind., Ném., IV, 56a Drachmann; schol. Lycophron, Alex., 310. Photios et la Souda, s.v. 'Iv $\gamma \xi$, prétendent qu'elle fut transformée en pierre. D'après

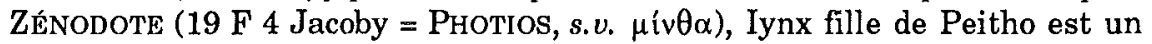
autre nom de Mintha qui menaça dans les Enfers le couple Hadès-Perséphone. Sur ce point, cf. M. DETIENNE, Les jardins d'Adonis, op. cit. , p. 139-184.

35 Principe mis en évidence notamment par J. RUDHARDT, op. cit. 
procédés magiques ${ }^{36}$. Néanmoins, les interprètes doivent avoir ainsi manifesté le souci d'inscrire les malheurs d'Iynx dans une séquence mythique significative. Dans cette hypothèse, il convient de rappeler qu'Io est l'ancêtre des Danaïdes dont les multiples aventures constituent un discours mythique complexe sur le mariage ${ }^{37}$, immuablement garanti par le couple olympien Zeus-Héra. Dès lors, que ce soit Io ou Iynx qu'ait désiré Zeus ensorcelé, le récit parle à la fois du désir contraignant $^{38}$ et de la légitimité du mariage.

Iynx a donc menacé le couple olympien et la punition n'a pas tardé. Un autre personnage a lui aussi attenté au paradigme divin de l'union matrimoniale. Ixion, fils de Phlégyas, a par deux fois été à l'encontre des règles qui président aux mariages. Recevant la fille d'Eioneus pour épouse, Ixion promet à son beau-père de nombreux cadeaux qu'il s'empresse de ne pas lui donner. Devant la colère d'Eioneus, il l'invite chez lui pour une conciliation et n'hésite pas à l'attirer dans un piège qui cause sa mort ${ }^{39}$. Premier humain à avoir versé le sang d'un parent ${ }^{40}$, Ixion ne trouva personne pour le purifier d'un tel crime. Zeus finit par accepter et lui offrit même l'hospitalité. Accueilli dans l'Olympe, Ixion s'éprend d'Héra et entreprend de s'unir à elle. Zeus suscite alors une nuée à l'image de son épouse, dont l'apparence trompeuse conduit Ixion à une union "sans les Charites» 41 . De cette union contre nature et contre les lois de la société naît un monstre, ancêtre des Centaures. Le châtiment est exemplaire : attaché par le Cronide sur une roue ailée suspendue dans les airs, Ixion répète aux mortels : "montrez à un bienfaiteur votre gratitude en lui offrant le contre-don qui remplit de joie» 42 .

36 S. EiTREM, art. Io, in $R E$, IX (1916), c. 1732-1743.

37 Esch., Suppl., 162-175; 274-323 : selon les termes mêmes des Danaïdes, leurs mésaventures résultent toujours de la colère d'Héra contre Io. Cf. M. DetienNe, Les Danaides entre elles. Une violence fondatrice de mariage, in L'écriture d'Orphée, Paris, 1989, p. 40-57.

38 Le violence dont les Égyptiades entendent user à l'égard des Danaïdes est plus brutale mais guère moins contraignante que les sortilèges magiques.

39 Dion. Sic., IV, 69, 3-4.

40 PIND., Pyth., II, 56-58.

41 Pind., Pyth., II, 78; A poliod., Epitome, I, 20; Diod. SIC., IV, 69, 4-5.

42 PIND., Pyth., II, 41-45 (trad. M. DETIENNE, op. cit., p. 167). 
La colère d'Héra à l'encontre d'Iynx avait fait de la nymphe un oiseau aux mouvements incessants, symbolisé pour les mortels par une roue utilisée dans des pratiques marginales. Ixion, par la colère de Zeus, devient une sorte d'iynx à mesure humaine ${ }^{43}$. Le contexte $d u$ symbole est dans les deux cas la mise en cause du couple par excellence que constituent Zeus et Héra, sans que l'initiative de l'adultère revienne à l'un des deux protagonistes divins. Si, comme Plutarque l'affirme, la charis est bien "la réponse de la femme au désir de l'homme" 44 , Ixion a bafoué cette donnée fondamentale de la relation entre les sexes, tout autant qu'Iynx qui avait ôté par ses sortilèges le libre-arbitre à son partenaire masculin.

\section{Des cultes civiques aux artifices de sorcières}

La relation entre homme et femme au cœur de la cité grecque est patronnée par toute une série de divinités masculines et féminines dont les rôles se complètent. L'assistance aux jeunes époux, notamment, concerne à des titres divers Zeus, Héra, Artémis, Peitho, Aphrodite ${ }^{45}$. Chaque cité comptait un ou plusieurs cultes que les jeunes mariés se devaient d'honorer avant et après leurs noces ${ }^{46}$. La nécessité d'un passage harmonieux du statut de jeune fille à celui d'épouse était ressentie par les législateurs qui n'hésitaient pas à intégrer l'une autre prescription à ce sujet dans leur œuvre d'ordonnancement ${ }^{47}$. La déesse d'Aphrodite ainsi que les personnifications de son cortège comme Peitho et les Charites étaient tout particulièrement chargées, avec Hermès, de conférer aux époux séduction et charme, à l'image des pouvoirs prêtés par la déesse à Héra dans l'Iliade et à Pandore chez

43 Comme l'a montré M. DetienNe (op. cit., p. 165), «Ixion se rattache aux valeurs de l'iynx".

44 Plut., Dialogue sur l'amour, 5 (Mor., 751d). Cf. J.-P. VERnANT, «HestiaHermès. Sur l'expression religieuse de l'espace et du mouvement chez les Grecs", in Mythe et pensée chez les Grecs. Etudes de psychologie historique, Paris, 1969, p. 104.

45 Plut., Quest. rom., 2 (Mor, 264b). Dans les Conseils aux fiancés, 138c-d, le même auteur associait les Muses et Aphrodite pour favoriser l'harmonie du mariage, et leur adjoignait Hermès car sle plaisir est indissociable de la raison", de même que Peitho et les Charites doivent permettre d'éviter les querelles.

46 Cf. V. Pirenne-Delforge, L'Aphrodite grecque. Contribution à l'étude des cultes et de...

47 L'exemple le plus clair est celui de Solon: PLuT., Vie de Solon, 20, 4. 
Hésiode, quelles qu'en soient les ambiguïtés. Dans un tel contexte, le rôle des dieux est intégré au bon fonctionnement d'une institution sociale fondamentale.

Les pratiques magiques, davantage que les rites d'une religion institutionnalisée, répondent à des besoins irrationnels et se fondent sur des forces impulsives. La magie est le lieu d'une référence à la nature plus qu'à la cité et à son ordre, fût-il implicite ${ }^{48}$. On comprend dès lors que les sentiments amoureux et leur charge affective potentiellement destructrice aient été exploités dans ces pratiques marginales qui offraient un terrain productif et fécond. Mais la magie a besoin des dieux dans le secret de ses alcôves, ces mêmes dieux que l'on sollicite au grand jour dans le culte officiel. Dans le cadre des charmes amoureux, les limites entre la religion et la magie sont ténues et, $a$ priori, seul le caractère souvent marginal de la pratique magique peut constituer un indicateur différenciel.

On a vu à quel point le discours mythique faisait valoir le caractère coercitif de l'attirance amoureuse et du désir : le symbolisme des liens y est récurrent. Or la force du lien est un des principes essentiels de l'action magique dont le lien est l'incarnation ${ }^{49}$. Les modes d'intervention d'Aphrodite et de ses suivants peuvent donc être qualifiés de "magiques", tout comme le ruban brodé $\pi$ orkî̀os. Où se situe dès lors la spécificité des sortilèges utilisés par la magicienne Simaitha décrite dans la IIe Idylle de Théocrite ? Ce que l'on a appris sur l'iynx pourrait permettre d'amorcer le tracé d'une délimitation entre les domaines. En effet, jamais Iynx, ni l'instrument censé porter son nom, n'apparaissent dans un autre contexte que celui de la magie amoureuse. Or, si Aphrodite est bien l'instigatrice de la roue du délire et si, en filigrane,

48 Sur ce sujet, on lira les belles pages introductives du livre d'André BERNAND, Sorciers grecs, Paris, Fayard, 1991. Néanmoins, des pratiques que l'on pourrait aussi qualifier de "magiques" intervenaient au cœur de la cité et même à l'initiative de la cité : cf. F. GRAF, An Oracle against Pestilence from a Western Anatolian Town, in $Z P E, 92$ (1992), p. 267-279. La question de la délimitation des domaines reste entière...

49 Cf. M. ElAADE, Le «dieu lieur» et le symbolisme des noeuds, in RHR, 134 (1948), p. 5-36 (repris dans Images et symboles. Essais sur le symbolisme magicoreligieux, Paris, 1952); J. ANNEQUIN, Recherches sur l'action magique et ses représentations (Ier et Ilèmes après J.C.), Paris, 1973 (Annales littéraires de l'Université de Besançon, 146. Centre de Recherches d'Histoire ancienne, 8), p. 19-21; A. BERNAND, op. cit. , p. 75-79. 
elle n'est pas étrangère à l'initiative malheureuse de l'effrontée $\mathrm{Iynx}^{50}$, on assiste, tant sur le plan mythique que sur le terrain des pratiques, à la manifestation extrême des pouvoirs de la déesse, manifestation que l'on pourrait de surcroît qualifier d'«a-sociale» puisque la réalisation de la volonté de l'un des partenaires résulte d'une forme de violence faite à l'autre. On saisit dès lors toute l'ambiguïté des pouvoirs d'Aphrodite dont les modes d'intervention habituels - on pourrait presque dire institutionnalisés - sont symbolisés, dans le mythe, par des représentations qui pourraient sans peine appartenir au monde de la magie. Néanmoins, quels que soient les liens de la séduction tels que les évoquent Homère dans l'Iliade ou Hésiode dans Les Travaux et les Jours, il s'agit de l'exercice équilibré et pleinement assumé de la sexualité divine au sein du couple Zeus-Héra et de la sexualité humaine au sein du couple à venir Pandore-Épiméthée. Point de sortilèges et d'interventions en vue d'annihiler la volonté de l'autre, point de contestation non plus de la légitimité de l'union matrimoniale.

La frontière entre religion et magie esquissée par A. Bernand n'est dès lors pas sans intérêt dans le cas des sortilèges amoureux : le rituel religieux est fixé par les traditions et les décisions de la cité, et le second relève de la fantaisie de l'individu ${ }^{51}$. Les dieux interviennent dans les deux cas, mais, en ce qui concerne Aphrodite, l'ancrage de ses prérogatives dans le vécu sensible et affectif des individus rend les frontières particulièrement perméables. À moins qu'elles n'existent pas.

Université de Liège

Vinciane PIRENNE-DELFORGE

Chargée de recherches au F.N.R.S.

Centre d'Histoire des Religions

Place du XX-Août, 32

B - 4000 LIÈGE

50 Photios et la Souda, s. $v$. "I $v \gamma \xi$.

51 A. BERNAND, op. cit. , p. 69. 